\title{
Bilateral Charcot's arthropathy of shoulder secondary to syringomyelia -a rare case report
}

\author{
Surojit Mondal ${ }^{1}$, Aniket Chowdhury ${ }^{2}$, Ranadeb Bandyopadhyay ${ }^{3}$, Rakesh \\ Kumar $^{4}$, Subrata $\mathrm{Pal}^{5}$, Eashin Gazi \\ Department of Orthopaedics, B.S.Medical College, West Bengal, India ${ }^{1,3,}$ \\ Department of Community Medicine, B.S.Medical College, West Bengal, India ${ }^{2,6,4}$ \\ Department of Pathology, B.S.Medical College, West Bengal, India ${ }^{5}$
}

\begin{abstract}
Neuropathic arthropathy of the shoulder is a rare disorder that has been described in fewer than sixty patients in the world literature $1,3,4,7,10,12,14,17,18,20,27$. In 1833, Mitchell presented clinical evidence of connection between so-called translatable rheumatism affecting the peripheral joints and disease of the spine but made no mention of dysfunction of the sensory or motor nerves (apart from the case of one patient who had an incomplete spinal-cord injury in association with an injury of the cervical spine). Thirty-five years after Mitchell's report, Charcot brought attention to the entity that now

bears his name ${ }^{3}$.

Neuropathic arthropathy, also known as Charcot joint, is a destructive arthropathy resulting from loss or diminution of proprioception, pain and temperature perception. ${ }^{12},{ }^{13,}{ }^{14}$ Patients with diabetes mellitus, syringomyelia and syphilis are particularly prone to this disease. ${ }^{15}$ Diabetic patients tend to have involvement of foot and ankle, while knee is the common site in syphilis. Patients with syringomyelia commonly suffer with shoulder and elbow involvement. ${ }^{16}$ till date only three cases of bilateral Charcot's arthropathy of shoulder were reported. Herein, we report a case that is felt to be of interest because of bilateral involvement with unusually late presentation)
\end{abstract}

\section{Case Report}

A 66-year-old male presented to our outpatient department with painless swelling of both his shoulders and restricted movements of the bilateral shoulder joints. His condition began when he was approximately 55 years old and he started experiencing reduced sensation in the bilateral upper limbs. Gradually he started experiencing reduced sensations in the bilateral lower limbs. Despite that, he did not seek medical attention and his condition gradually worsened. There was no history of significant trauma in the past.

Physical examination revealed generalized swelling about the shoulder, more on the right side [Figure 1]. On the right side, there was abnormal motion, where the shoulder joint would be expected, with huge abnormal swelling. On the other hand, the left shoulder joint was found to be dislocated, with humeral head anterior to glenoid cavity, leading to significant restricted joint movement. On palpation, the shoulder joints were nontender. Bilateral shoulder movements were restricted. Active forward flexion was $80^{\circ}$ on the right and $30^{\circ}$ on the left side, abduction $20^{\circ}$ on the left and $25^{\circ}$ on the right side and internal rotation bilaterally up to the sacrum. He had 4/5 shoulder abductor strength and 4/5 shoulder flexor and extensor strength bilaterally. Range of motion for passive movements was significantly higher than on active movements, more on the right side. Passive movement was painful on terminal range of motions. His biceps strength was $4 / 5$; triceps strength was $5 / 5$ while motor strength in bilateral distal extremities was $5 / 5$. There was decreased sensation involving the entire upper extremities bilaterally. The biceps, triceps and brachioradialis reflex were absent. 


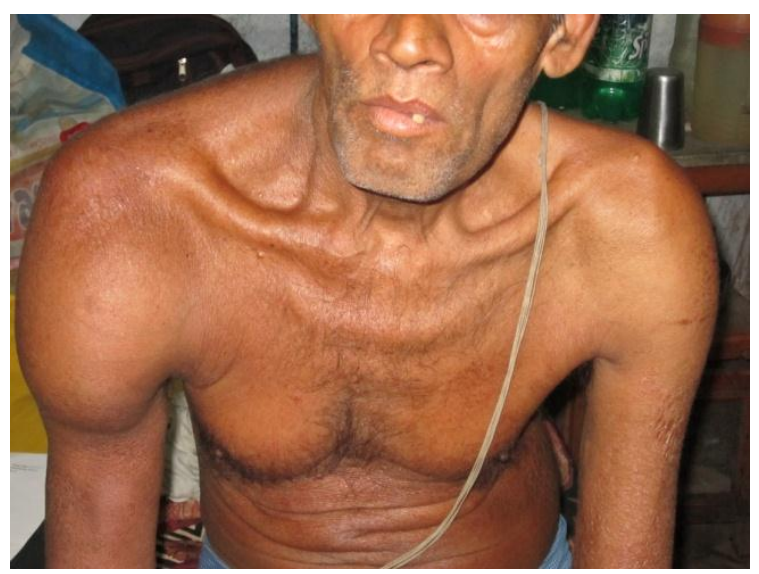

Figure 1: Clinical photo of the patient showing involvement of bilateral shoulder joint

Patient was worked up and X-ray, complete blood counts, erythrocyte sedimentation rate, fasting blood sugar, venereal disease research laboratory test, level of vitamin B12, electromyography (EMG) and nerve conduction velocity of both upper limbs, measurement of the crystal for gout and pseudo gout, magnetic resonance imaging

(MRI) of cervical and thoracic spine and abdominal ultrasonography were performed. Although the overlying skin temperature was normal, still joint aspiration was performed to rule out the remote possibilities of an infected joint. No organism grew on culture. . An incisional biopsy was performed, and gram stains and routine cultures were negative. The biopsy revealed necrotic bone, hypervascularity, and degenerative articular cartilage Other than radiography and MRI; all the haematological investigations were within normal limits, on the basis of which syphilis and diabetes were ruled out. A purified protein derivative test was nonreactive. X-ray of right shoulder showed destruction of humeral head with fragmentation of accromian process and lateral end of clavicle, which falls into the productive variant of Charcot shoulder [Figure 2]. X-ray of the left shoulder showed anterior dislocation of shoulder with diffuse destruction of proximal humerus, acromian process and lateral end of clavicle and heterotopic new bone formation [Figure 3]. MRI (magnetic resonance imaging) of cervical and dorsal spine showed large syrinx from the $3^{\text {rd }}$ cervical to the $5^{\text {th }}$ cervical spine [Figure 4]. At that time, various modalities of treatment were considered. Limb elevation, shoulder abduction brace and nonsteroidal anti-inflammatory drugs were advised. His swelling gradually subsided after approximately 4 weeks of conservative treatment. As instability and not pain was the chief complaint, a custom-made shoulder abduction brace was advised on both the sides and underwent intensive shoulder rehabilitation programme. After that, the patient returned monthly and remained asymptomatic for the next 1 year.

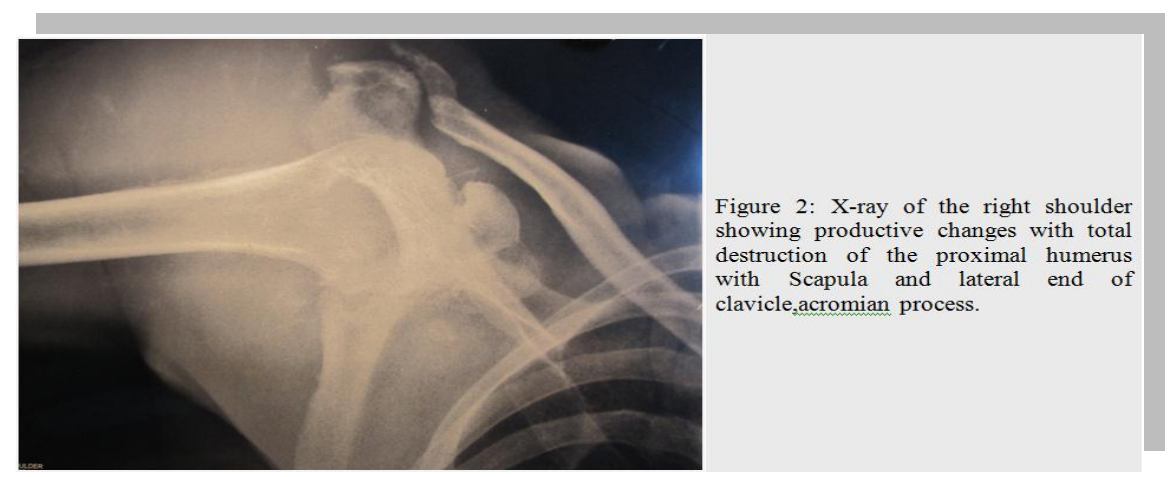



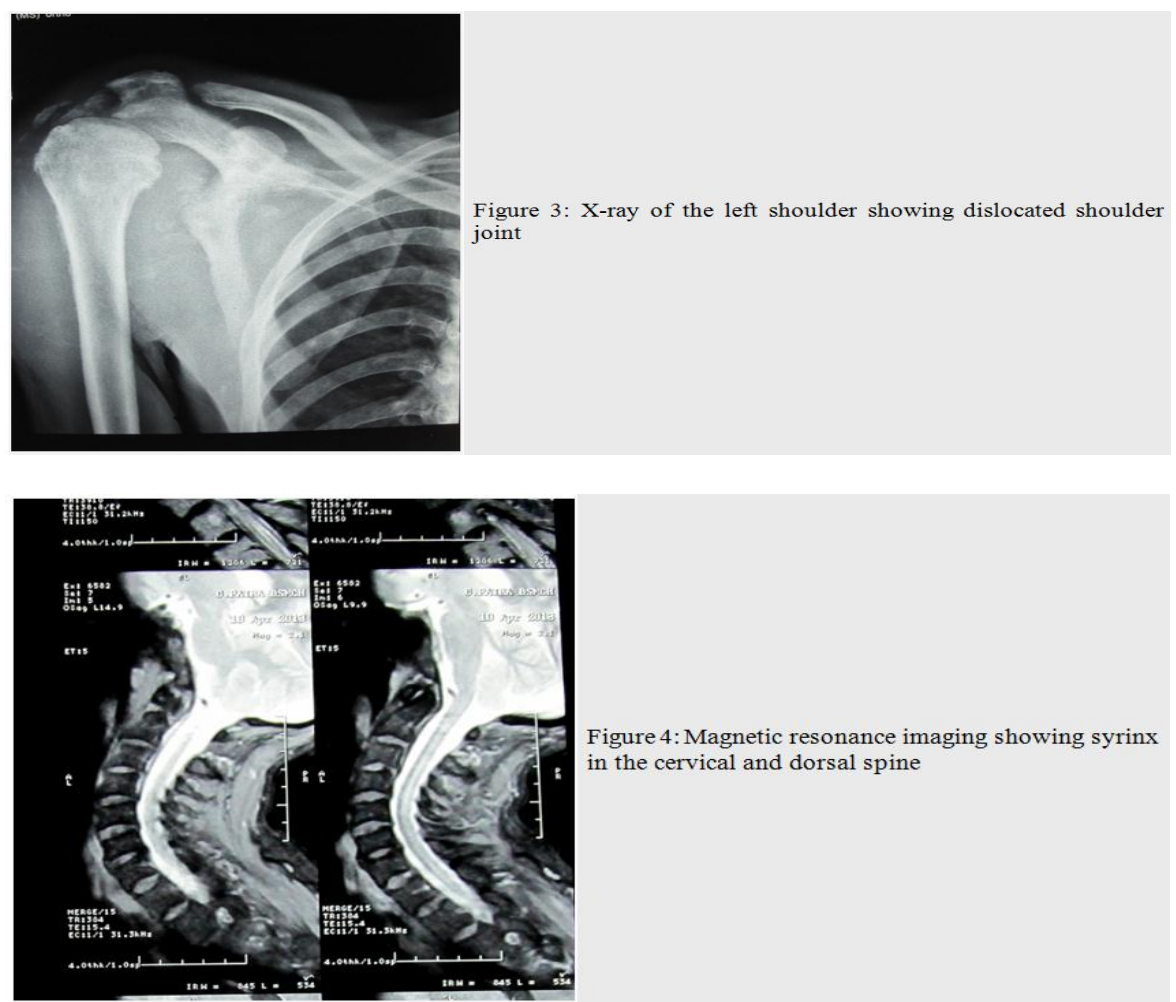

Figure 4: Magnetic resonance imaging showing syrinx in the cervical and dorsal spine

\section{Discussion}

Charcot arthropathy is located in the shoulder in only 5\% cases. Meyer et al. Stated that arthropathies develop in $25 \%$ of the cases of syringomyelia, and that $80 \%$ of syringomyelia arthropathies involve the upper limb. (Exact numbers were not reported in that study. $)^{17}$ there are two theories describing the pathogenesis of neuropathic osteoarthropathy. These are the neurotraumatic and the neurovascular theories. The neurotraumatic theory, first described by Johnson in 1967, involves repetitive trauma sustained by an insensate joint. The neurovascular theory, proposed by Allman and colleagues, describes active bone resorption by osteoclasts secondary to sympathetic dysfunction and a neurally mediated persistent hyperaemia. If fractures and other forms of trauma are involved, this theory suggests that they occur secondarily. ${ }^{15,17}$

Neuropathic arthropathy of the shoulder often presents in a striking fashion, producing extensive and rapid destruction of the proximal aspect of the humerus and the glenoid. The differential diagnosis often includes primary and metastatic malignant tumour, tuberculous and microbial infection, and Gorham disease (vanishing bone disease). Syringomyelia is a disorder involving a fluid-containing cavity (syrinx) within the spinal cord. These cavities commonly occur in the lower cervical and upper thoracic segments, and the distension may propagate proximally. Its causes include congenital, traumatic, infectious, degenerative, and vascular or tumour related. ${ }^{13,14,18,19}$ MRI is considered the gold standard for visualization of a syrinx.

Syringomyelia is a potential cause of neuropathic osteoarthropathy of the shoulder, or "Charcot's shoulder." the work-up of a patient with shoulder dysfunction should include a thorough history and physical examination and radiographic plain films. Other pertinent investigations such as CBC (complete blood counts), ESR (erythrocyte sedimentation rate), FBS (fasting blood sugar), VDRL (venereal disease research laboratory test), level of vitamin b12, EMG (electromyography) and nerve conduction velocity of both upper limbs, measurement of the crystal for gout and pseudo gout and abdominal Ultrasonography should be performed. Once a neuropathic joint has been diagnosed, its aetiology should be pursued with aseptic joint aspiration to look for infection or tumour, and an MRI to evaluate for syringomyelia if the aetiology remains in doubt. Syringomyelia may present as instability of the shoulder, and instability or frank dislocation is not unusual in the neuropathic shoulder.

Previous studies reported failure when arthrodesis was performed to treat neuropathic arthropathy of the shoulder, and concluded that this condition is a contraindication to arthrodesis. ${ }^{14,} 20$ Those authors also stated that synovectomy is not helpful and that a neuropathic shoulder should be treated nonoperatively, with an emphasis on the maintenance of function. The therapy for neuropathic arthropathy is conservative. Preventing trauma to the joint with proper splinting is key to treatment. Aspiration of large effusions and splinting will prevent further ligamentous laxity. ${ }^{12,}$ 
we concur with these conclusions and agree that the maintenance of function, rather than immobilization, is the keystone of treatment of neuropathic arthropathy. We managed our patient conservatively, as suggested by the majority of the literature, and the patient was satisfied with the treatment. To our knowledge, this is among few case report of bilateral Charcot's arthropathy of the shoulders from the Indian subcontinent. It is hoped that this case report will increase awareness of physicians about this destructive joint disease.

\section{References}

[1] Brailsford, J. E: Serial radiographic appearances of a neuropathic shoulder-joint. British J. Surg., 22:424-427, 1935.

[2] Charcot, J.-M.: Sur quelques arthropathies qui paraissent dependre d'une lesion du cerveau ou de la moelle epiniere. Arch, physiol. norm, pathol, 1:161-178, 1868

[3] Drvaric, D. M.; Rooks, M. D.; Bishop, A.; and Jacobs, L. H.: Neuropathic arthropathy of the shoulder. A case report. Orthopedics, 11:301-304, 1988 .

[4] Goodman, M. A., and Swartz, W.: Infection in a Charcot joint. A case report. J. Bone and Joint Surg., 67-A: 642-643, April 1985.

[5] Heylen, Y.: Neuropathic arthropathy of the shoulder secondary to syringomyelia. J. Beige Radiol, 76:232-233, 1993.

[6] Knur, E.: Two cases of Charcot's shoulder arthropathy. Acta Orthop. Scandinavica, 58:581-583, 1987.

[7] Meyer, G. A.; Stein, J.; and Poppel, M. H.: Rapid osseous changes in syringomyelia. Radiology, 69:415-418, 1957.

[8] Parikh, J. R.; Houpt, J. B.; Jacobs, S.; and Fernandes, R. J.: Charcot's arthropathy of the shoulder following intraarticular corticosteroid injections. / Rheumatol, 20: 885-887, 1993.

[9] Pendergrass, E. P.; Gammon, G. D.; and Powell, J. H.: Rapid development of bone changes in patient with syringomyelia as observed Roentgenologically. Radiology, 45:138-146, 1945.

[10] To that form caused by peripheral nerve disease or injury. J. Am. Med. Assn., 82: 27-29,1924.

[11] Rhoades, C. E.; Neff, J. R.; Rengachary, S. S.; Batnitzky, S.; Ketcherside, J.; Price, H. I.; and Jacobs, R. R.: Diagnosis of posttraumatic Syringohydromyelia presenting as neuropathic joints. Report of two cases and review of the literature. Clin. Orthop., 180:182-187,1983.

[12] Villiaumey, J.; Caron, J.-P.; Larget-Piet, B.; and Haddad, A.: Arthopathie destructive de l'epaule revelatrice d'un syndrome syringomyeliquechez un malade atteint d'une paraplegie post-traumatique. Rev. rhumat., 40: 277-280, 1973.

[13] Johnson JT. Neuropathic fractures and joint injuries: Pathogenesis and rationale of prevention and treatment. J Bone Joint Surg Am 1967; 49:1-30.

[14] Jones J, Wolf S. Neuropathic shoulder arthropathy (Charcot joint) associated with syringomyelia. Neurology 1998; 50:825-7.

[15] Mau H, Nebinger G. Die Schultergelenksarthropathie bei der Syringomyelie. Zeitschr Orthop 1986; 6:157-64.

[16] Allman RM, Brower AC, Kotlyarov EB. Neuropathic bone and joint disease. Radiol Clin North Am 1988; 26:1373-81.

[17] Oxford Textbook of Medicine. Vol. 2.3. Oxford: Oxford University press 1996. p. 1486, 1490, $2979,3006$.

[18] Harrison, Principals of Internal Medicine. Vol. 1,2. New York: McGraw Hill: 1998. p. 1027, $1733,1953,2076$.

[19] 18.Klekamp J. The pathophysiology of syringomyelia - historical overview and current concept. Acta Neurochir (Wien) 2002; 144:649-64.

[20] Meyer GA, Stein J, Poppel MH. Rapid osseous changes in syringomyelia. Radiology 1957; 69:415-8. ]

[21] Eichenholtz SN. Charcot joints. Thomas CC, editor. Springfield: 66. p. 11 